\title{
Contralateral Hyperhidrosis after Cerebellar Infarct and Ischemia in Vertebrobasilar Artery Territory: A Case Report
}

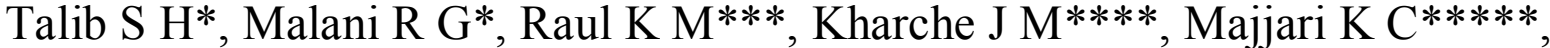 \\ Mannikar VA***** \\ *Professor \& Head Medicine, **Professor \& Head Radiology, ***Professor Medicine, ****Associate Professor \\ Medicine, *****Resident Medicine,* \\ Mahatma Gandhi Missions Medical College \& Hospital, Aurangabad.431003
}

\begin{abstract}
Hyperhidrosis from cerebral and cerebellar infarction has been reported in stroke literature with paucity. The pathophysiological mechanism of the same has remained obscure. Contralateral excessive sweating was noted in a 70 years old male patient 48 hours after the stroke over forehead, face and upper trunk. Clinical and MRI studies revealed Cerebellar infarct and Ischemia in Vertebrobasilar Artery Territory. No associated hypothalamic dysfunction, Horner's Syndrome or any other autonomic dysfunction were noted in the case. The phenomenon of contralateral hyperhidrosis could be attributed to lesion of the sympathetic pathway that controls sweating. This putative pathway is close to the corticospinal tracts in the brainstem (Vertebrobasilar Artery Territory).
\end{abstract}

Keywords: Hyperhidrosis, Cerebellar Infarct, Vertebrobasilar Artery Territory Ischemia.

\section{Case History}

Patient DA, 70 years/ gentleman, was apparently alright 1 day prior to hospitalization, when he experienced giddiness, nausea and had frequent episodes of vomiting early morning while getting up. An hour later he developed tingling, numbness on right side of the body associated with jerky movements which persisted for few minutes and slurred speech. MRI Brain revealed acute infarct in left cerebellar hemisphere involving both Superior Cerebellar Artery and Posterior Inferior Cerebellar Artery territories. Brainstem did not reveal any abnormality [image -1]. Next day morning patient was having altered mental state. Within a couple of hours patient's condition worsened; responding only to noxious stimuli. He developed tetraplegia with bilateral Babinski sign and alteration in respiratory pattern. Right side pupil was smaller and left side pupil was semi-dilated; both having sluggish light reflex with corneal and oculocephalic reflexes absent. Patient had no ptosis. An endotracheal tube was inserted and patient was mechanically ventilated. A couple of hours later excessive hyperhidrosis on right side of face, arm and chest was noted with large drops of sweat dripping continuously from his forehead and chest [image -2]. Patient was afebrile. ECG was showing sinus Bradycardia with a Heart rate of 52/minutes. No Electrolyte abnormalities reported. MRI could not be repeated due to poor condition of the patient. Hyperhidrosis of right face, neck, and chest persisted until his death on the $4^{\text {th }}$ day of hospitalization. Postmortem was denied.

\section{Discussion}

Hyperhidrosis is reported in varied lesions of central nervous system. It is noted in Tetraplegia, spinal cord trauma and high level paraplegia ${ }^{[1]}$. Excessive sweating has also been described in hemispheric stroke especially when opercular cortex is involved ${ }^{[2]}$. However hyperhidrosis is restricted to the contralateral side of the body. Contralateral hyperhidrosis may be observed in late phase of Lateral Medullary Infarct and is likely due to lesions of sympathetic pathway passing through lateral medulla ${ }^{[3]}$. Excessive sweating from cerebral infarct ${ }^{[2,4]}$, hypothalamus ${ }^{[5]}$, Pons ${ }^{[6,7]}$, and spinal cord $^{[8]}$ is also reported in the literature. Excessive sweating as an uncommon sign of basilar artery occlusion is described by Awada et al ${ }^{[7]}$. Contralateral hyperhidrosis after cerebellar infarct and bilateral pontine infarct is described by Kim BS and associates ${ }^{[9]}$.

In the present case, features point towards a pontine lesion as this patient had giddiness, dysarthria, rapid clonic jerky movements, rapidly developing confusion, and absent corneal and oculocephalic reflexes. The findings remain consistent with occlusion of Basilar Artery. The pathophysiological mechanism of unilateral hyperhidrosis in cerebellar infarct is still unclear. This phenomenon could be due to disruption of putative inhibitory neural pathway that controls sweating of contralateral face and body. At the level of pons and medulla, the fibers descend in close proximity to corticospinal tracts and along inner side of facilitatory fibers which are speculated to travel through dorsolateral part of pontine tegmentum. MRI Brain was suggestive of acute infarct in left cerebellar hemisphere with involvement of Superior Cerebellar Artery and Posterior Inferior Cerebellar Artery territories. Perfusion imaging was not performed, hence suspected brainstem ischemia was not 
picked up on imaging. Superior Cerebellar arteries from the Basilar Artery, supply the lateral aspect of Pons and Mid-brain together with superior surface of Cerebellum. Pons is supplied by small and large branches of Basilar Artery. These important points need to be considered when assessing the case clinically. The most common vascular disease affecting the vertebrobasilar system is atherosclerosis. The atherosclerosis often produces stenosis at the origins of both vertebral arteries to cause Vertebrobasilar Ischemia. It is also to be noted that embolic occlusion of the Vertebrobasilar system is uncommon and emboli are typically from Aortic Arch, Subclavian Arteries \& Vertebral Arteries and may occlude the Basilar artery resulting in Vertebrobasilar insufficiency. Even with Vertebral artery occlusion and the basilar inadequacy the collaterals (Circle of Willis) may prevent ischemia. However Circle of Willis is intact in only $50 \%$ of the patients ${ }^{[10]}$. Patient's condition deteriorated fast and MR Angiography could not be done. Patient expired on the $4^{\text {th }}$ day of hospitalization. Syndrome of Brainstem compression by swollen Cerebellar infarct in combination with Basilar Artery Ischemia remains another postulation ${ }^{[11]}$.

\section{References:}

[1]. Appenzeller O. The autonomic nervous system. Amsterdam: Elsevier, 1982.

[2]. Labar DR, Mohr JP, Nichols FT, Tatemichi TK. Unilateral hyperhidrosis after cerebral infarction. Neurology 1988; 38: 1679-82.

[3]. Rousseaux M, Hurtevent JF, Benaim C, Cassim F. Late contralateral hyperhidrosis in lateral medullary infarct. American Heart Association Journal. Stroke. 1996; 27: 991-95.

[4]. Korpelainen JT, Sotaniemi KA, Myllyla VV. Asymmetric sweating in stroke: a prospective quantitative study of patients with hemispheral brain infarction. Neurology 1993; 43:1211-14.

[5]. Sakashita Y, Kakuta K, Kakuma K, Matsuda H. Unilateral persistent hyperhidrosis after ischemic stroke [in Japanese]. Rinsho Shinkeigaku. 1992; 32:454-56.

[6]. Fisher CM. Bilateral occlusion of basilar artery branches. J Neurol Neurosurg Psychiatry. 1977; 40:1182-89.

[7]. Awada A, Ammar A, Al-Rajeh Saad, Borollosi M. Excessive sweating: an uncommon sign of basilar artery occlusion. J Neurol Neurosurg Psychiatry. 1991; 54:277-78.

[8]. Kneisley LW. Hyperhidrosis in paraplegia. Arch Neurol. 1977; 34:536-39.

[9]. Kim BS, Kim YI, Lee KS. Contralateral hyperhidrosis after cerebral infarction. American Heart Association Journal. Stroke. 1995; 26: 896-99.

[10]. Kumral E, Kisabay A, Atac C. Lesion patterns and etiology of ischemia in the anterior inferior cerebellar artery territory involvement: a clinical - diffusion weighted - MRI study. Eur J Neurol. 2006 Apr; 13(4): 395-401.

[11]. Sinha KK. Brain Stem Infarction: Clinical Clues to Localise Them. Journal, Indian Academy of Clinical Medicine 2000; 1 (3): 213 21.

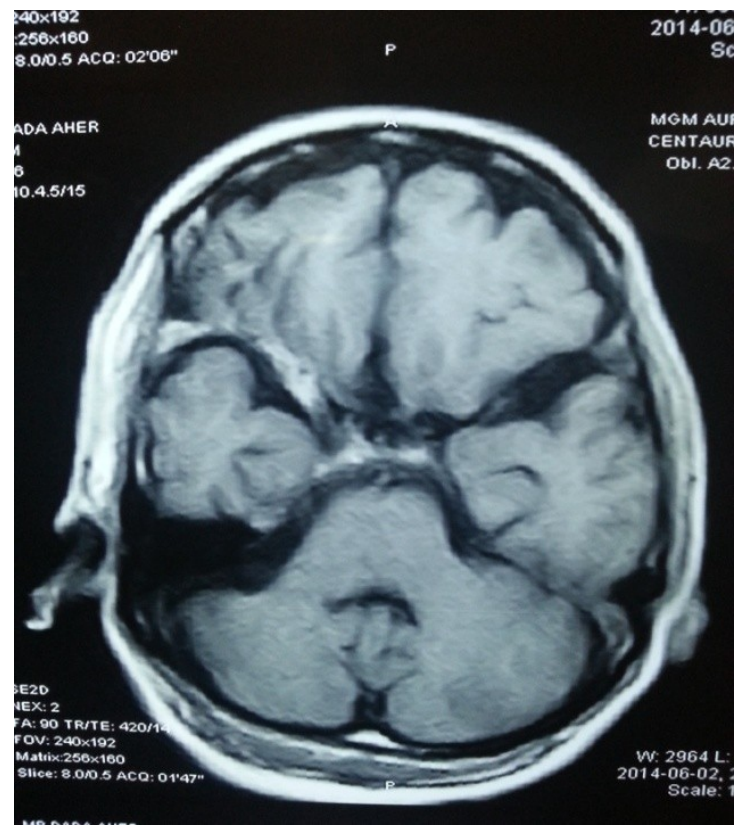

Image - 1: MRI Brain showing acute infarct in left cerebellar hemisphere involving both Superior Cerebellar and Posterior Inferior Cerebellar Artery territories. Brainstem does not reveal any abnormality. 


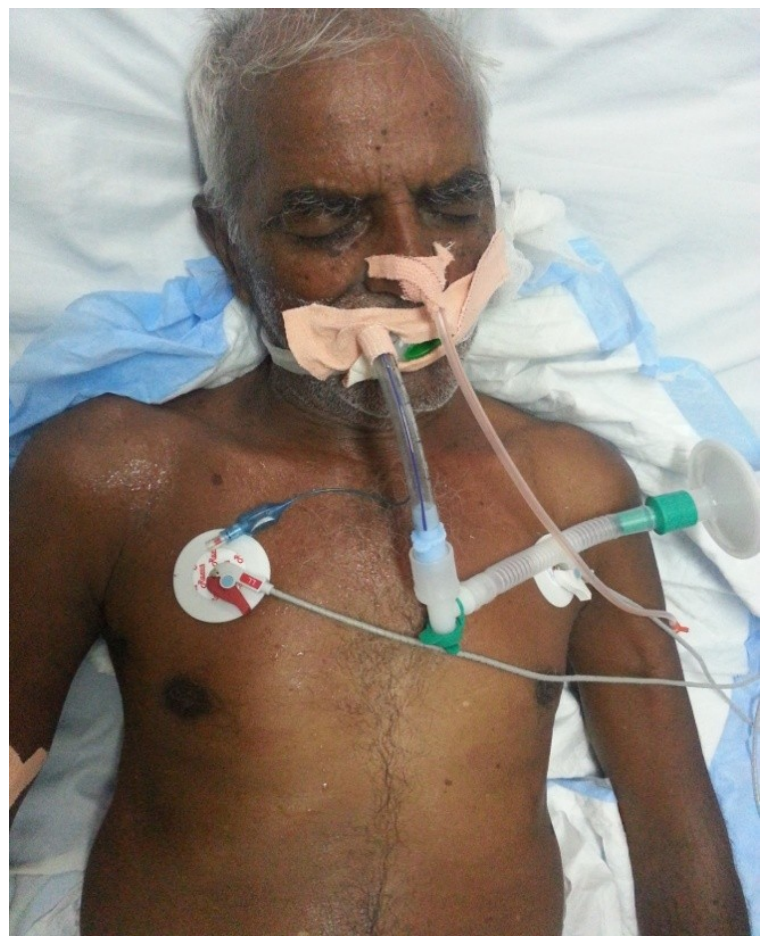

Image -2: Excessive hyperhidrosis seen on right side of face, arm and chest. 